\title{
Mescaline : la religion du peyotl
}

\section{Mescaline : the religious peyote cactus}

Pascal KINTZ

Institut de Médecine Légale, 11, rue Humann - 67000 STRASBOURG

Tél : +33 390243349 - Fax : +33 390243362 - E-mail : pascal.kintz@wanadoo.fr

(Reçu le 8 janvier 2004 ; accepté le 30 janvier 2004)

\section{$\boldsymbol{R E} \boldsymbol{E} \boldsymbol{S U E}$}

Obtenue à partir du peyotl, cactus des zones arides du Mexique ou du sud-ouest des Etats-Unis, référencé comme Lophora williamsii ou Anhalonium Lewinii, la mescaline est un hallucinogène de puissance faible. Elle est principalement contenue dans les excroissances du sommet du cactus, dénommées boutons de peyotl.

Les Indiens mangent la couronne crue, séchée et écrasée en purée. Parfois, selon les rites religieux, la consommation peut se faire en décoction ou après incision, grillée.

La dose hallucinogène de la mescaline est de l'ordre de 200 à $500 \mathrm{mg}$, soit une activité 4000 fois plus faible que celle du $L S D$, composé de référence. Les effets sont rapides, dans l'heure qui suit la prise, et persistent environ 12 heures. Ils sont caractérisés par une altération de la perception et de l'humeur, des troubles de la vigilance, de la désorientation et des perturbations de la mémoire immédiate. Les effets secondaires sont de type nausées, vomissements, anxiété pouvant évoluer vers la paranoïa.

Sur le plan analytique, la mescaline est identifiable et dosable selon les procédures classiques pour les amphétamines : extraction en milieu alcalin par l'acétate d'éthyle en présence de mescaline-d, dérivation par HFBA, identification par chromatographie en phase gazeuse couplée à la spectrométrie de masse par les ions m/z 181, 194 et 407.

La littérature ne mentionne pas de cas de décès directement imputable au principe actif. Néanmoins des observations de défénestration ou des homicides sous influence de mescaline ont pu être rapportées. Par ailleurs, la conduite automobile sous mescaline peut provoquer des accidents mortels. La mescaline et le peyotl sont dans nos sociétés européennes des sujets de curiosité plus que d'inquiétude, et leur usage reste localisé en Amérique du Nord.

\section{MOTS-CLÉS}

Mescaline, hallucinogène, cactus, peyotl, urine.

\section{SUMMARY}

Mescaline, or 3,4,5-trimethoxyphenethylamine, is one of the most widely studied of the hallucinogens as well as one of the weakest, by volume. Quantitatively, mescaline serves as a sort of standard among hallucinogens. It is a naturally occuring alkaloid, found in the peyote cactus, that grows singly or in clusters in Mexico and the south-western United States. The dried tops of the plants, known as peyote buttons, have been used by Indian shamans for centuries.

Typical hallucinogenic doses range from 200 to $500 \mathrm{mg}$, with blood concentrations of $3.8 \mathrm{mg} / \mathrm{l}$ at $2 \mathrm{~h}$ and $1.5 \mathrm{mg} / \mathrm{l}$ at $7 \mathrm{~h}$ after ingestion.

On an analytical point, testing for mescaline is not more difficult than testing for amphetamine, as the drug can be extracted and derivatizated by the same procedures. After HFBA derivatization, mescaline target ions include $\mathrm{m} / \mathrm{z} 181,194$ and 407.

Lethal overdoses of mescaline have never been reported, nor have there been any reports of medical complications associated with its use. The deaths that have been reported have been accidental, usually as a result of drug-induced confusion.

\section{KEY-WORDS}

Mescaline, peyote buttons, hallucinogens, urinalysis. 
Le peyotl a été un sujet de controverse depuis l'arrivée en Amérique des conquérants espagnols. Il a donné lieu à des persécutions et son usage a été l'objet de bien des tentatives de suppression. On peut considérer ce cactus comme le prototype des hallucinogènes du Nouveau Monde. A ce titre, la mescaline constitue la substance de référence dans toute tentative de classification.

\section{Botanique et chimie}

La mescaline ou 3,4,5-trimethoxy-B-phénéthylamine (Figure 1) est un alcaloïde hallucinogène, isolé la première fois en 1896 (par Hefter) du peyotl ou peyote (Lophora williamsi ou Anhalonium lewinii), un cactus sans épine qui pousse au sud ouest des Etats Unis et au Mexique dans le désert ou les régions de montagne. La plante est de couleur vert-bleu, de 2 à $7 \mathrm{~cm}$ de haut pour 4 à $12 \mathrm{~cm}$ de diamètre. Le cactus pousse seul ou en groupe. Le dessus de la plante, appelé également couronne, est vendu sous la dénomination bouton de peyotl (peyotl buttons). Dans la pratique, les sommités sont coupées en rondelles puis séchées.

La mescaline représente environ $6 \%$ de la plante. L'extraction de la mescaline à partir de la plante est aisée. Elle fait presque toujours appel au protocole suivant : la partie supérieure de la plante (couronne) est mise à sécher au soleil (la mescaline est stable à la chaleur et aux ultra-violets, et non-volatile), puis pulvérisée, mélangée avec du méthanol pendant un jour, filtrée puis enfin extraite par du chloroforme. La synthèse chimique est longue et difficile (1).

Au moins 60 alcaloïdes ont été identifiés dans le peyotl, appartenant essentiellement à 2 groupes, les B-phénethylamines (mescaline) et les tetrahydroisoquinoléines. Tous ces composés diffèrent du LSD et des principaux hallucinogènes par l'absence d'une structure indolique. Deux autres homologues de la mescaline ont été identifiés dans le peyotl, la $\mathrm{N}$-acétyl-mescaline et la $\mathrm{N}$ méthyl-mescaline. L'activité hallucinogène de ces composés semble être très faible (1).

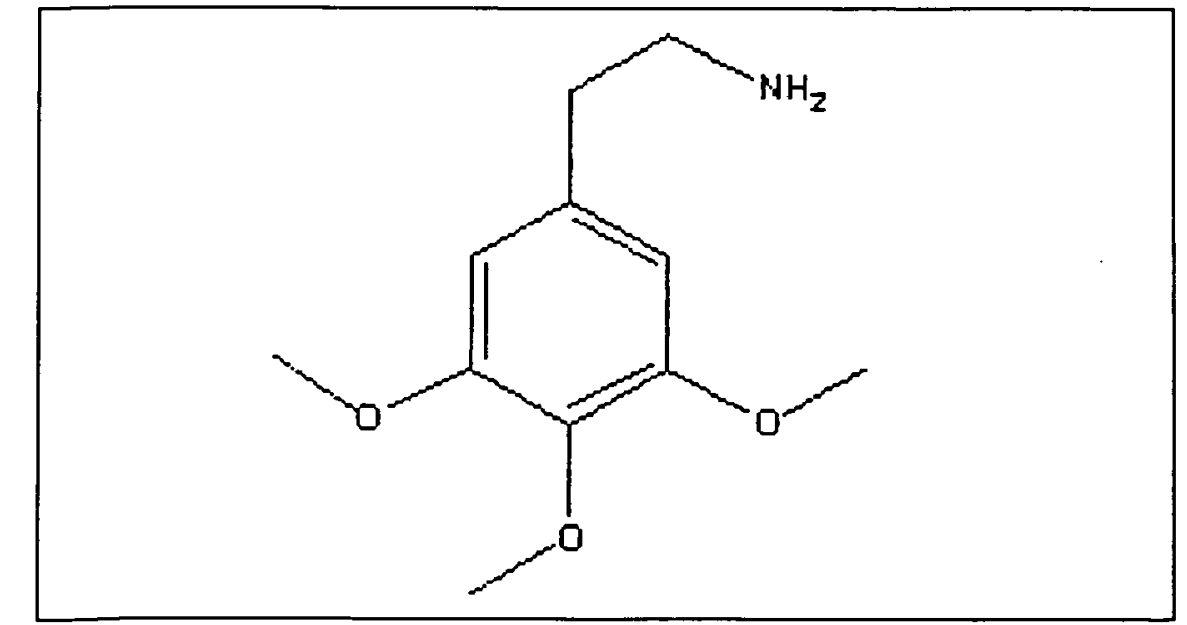

Figure 1 : Structure chimique de la mescaline.
La mescaline, de structure chimique $\mathrm{C}_{11} \mathrm{H}_{17} \mathrm{NO}_{3}$, a un poids moléculaire de 211,26.

Elle est modérément soluble dans l'eau, soluble dans l'alcool, le chloroforme ou le benzène et pratiquement insoluble dans l'éther di-éthylique ou l'éther de pétrole.

\section{Pharmacologie et toxicologie}

Le peyotl sert depuis des siècles aux cérémonies religieuses des Indiens du Mexique. Les extrémités de la plante ont été consommées par les chamans, puis au début du $19^{\text {eme }}$ siècle par les Apaches, les Kiowas et les Commanches qui la mâchait au début de leurs rituels religieux. Les Indiens mangent la couronne crue, séchée et écrasée en purée. Parfois, selon les rites religieux, la consommation peut se faire en décoction ou après incision, grillée. Lors d'une cérémonie, chaque Indien utilise de 4 à 30 têtes.

Au contraire, dans la rue, la mescaline est proposée sous forme cristalline, chlorhydrate ou sulfate, en comprimé, gélule, capsule gélatineuse, ou solution buvable. En Amérique du Nord, et plus particulièrement au Canada, $90 \%$ des échantillons présumés de mescaline, analysés dans les laboratoires de toxicologie, contenaient de la phencyclidine ou du LSD.

La dose hallucinogène de la mescaline est de l'ordre de 200 à $500 \mathrm{mg}$, soit une activité 4000 fois plus faible que celle du LSD, composé de référence. Les effets sont rapides, dans l'heure qui suit l'exposition, et persistent environ 12 heures. La demi-vie de la mescaline est de l'ordre de 6 heures (2). Une dose de $500 \mathrm{mg}$ conduit à des concentrations plasmatiques de 1 à $4 \mathrm{mg} / \mathrm{l}$.

Environ $60 \%$ de la dose est excrétée inchangée dans les urines, les autres métabolites étant surtout l'acide 3,4,5trimethoxyphenylacetique (27-30\%) et la N-acétyl-3,4dimethoxy-5-hydroxyphénéthylamine $(5 \%)(2,3)$.

La mescaline est un agoniste des récepteurs sérotoninergiques et dopaminergiques. La tolérance est faible, elle peut être croisée avec le LSD et la diméthyltryptamine. On ne note pas de dépendance physique, mais une dépendance psychique réelle.

La toxicité somatique de la mescaline est faible, sans commune mesure avec celle des composés de synthèse de type ecstasy.

La structure chimique de la mescaline peut être modifiée pour conduire à d'autres composés, mais seule l'addition d'un groupe méthyle sur le carbone en alpha conserve l'activité psycho-active. L'homologue exact de la mescaline est la TMA (2,4,5-trimethoxyamphetamine).

Comme tous les stimulants du système sympathique, la mescaline augmente la pression artérielle, la fréquence cardiaque et la température corporelle. Ces effets s'accompagnent souvent de crise d'angoisse, de tremblements, de nausées, voire de vomissements.

L'effet majeur de la mescaline est l'induction d'hallu- 
cinations visuelles, auditives et tactiles, avec distorsion du temps et de l'espace. Elle conduit également à une modification de l'image corporelle (perception erronée du corps), ce qui en a fait une substance testée en psychiatrie et sexologie. Des troubles de la vigilance et une altération de la mémoire immédiate ont également été rapportés.

La première phase de nausées semble être une constante. Shulgin et Shulgin dans leur livre culte PIHKAL (1) la décrivent également. Les auteurs rapportent plusieurs expériences avec 300,350 et $400 \mathrm{mg}$ de mescaline, dominées par des hallucinations visuelles et une modification de l'environnement, particulièrement auditif.

La secte Native American Church, aux Etats Unis et dans l'ouest du Canada, qui compte environ 250000 membres a obtenu l'autorisation officielle de consommer de la mescaline. Pendant les nuits de prières et de louanges, le peyotl est considéré comme un messager divin qui permet de communiquer avec Dieu, sans l'intermédiaire d'un prêtre.

\section{Aspects analytiques}

La mescaline est inscrite sur la liste 1 des produits stupéfiants, selon la convention de Vienne de 1962. C'est également un stupéfiant en regard de la législation française. Sa caractérisation est souvent effectuée à partir du cactus (4).

Afin de répondre à la demande de la justice, le laboratoire de toxicologie de l'IML de Strasbourg a développé une procédure analytique applicable aux urines.

Après optimisation, cette méthode peut également être utilisée pour le sang ou les cheveux (puisque la mescaline est stable en milieu alcalin).

La méthode est la suivante :

- $1 \mathrm{ml}$ d'urines

- 200 ng mescaline- $\mathrm{d}_{9}$

$-1 \mathrm{ml} \mathrm{NaOH} 1 \mathrm{~N}$

- $5 \mathrm{ml}$ acétate d'éthyle

Agitation $10 \mathrm{~min}$, centrifugation, recueil et évaporation de la phase organique

- dérivation par $50 \mu \mathrm{l} \mathrm{HFBA}+100 \mu \mathrm{l}$ acétate d'éthyle, $65^{\circ} \mathrm{C}$ pendant $20 \mathrm{~min}$

- évaporation, et reprise dans $50 \mu \mathrm{l}$ d'acétate d'éthyle - injection de $1 \mu \mathrm{l}$ dans un système de chromatographie en phase gazeuse couplée à la spectrométrie de masse.

La mescaline dérivée est caractérisée par les ions $\mathrm{m} / \mathrm{z}$ 181, 194, 226 et 407 (Figures 2 et 3). L'ion de quantification est le $\mathrm{m} / \mathrm{z} 407$, versus $\mathrm{m} / \mathrm{z} 416$ du standard interne.

La mescaline est éluée après tous les dérivés de type méthylènedioxyamphétamine, et donc aisément séparée des stimulants usuels.

La méthode est linéaire pour des concentrations de 0,1 à $50 \mathrm{mg} / \mathrm{l}$, avec $\mathrm{r}=0,998$. La limite de détection avec un rapport signal-sur-bruit $>3$ est de $10 \mu \mathrm{g} / \mathrm{l}$, soit une valeur largement inférieure aux concentrations usuelles. A $10 \mathrm{mg} / \mathrm{l}$, le coefficient d'extraction est de $81,2 \%$, pour une répétabilité $(\mathrm{n}=8)$ à $7,6 \%$.

\section{Aspects médico-légaux}

Aucun usage médical n'est communément accepté.

La littérature ne mentionne pas de cas de décès directement imputable au principe actif. La conduite automobile sous mescaline peut provoquer des accidents mortels (5). Dans le cas d'un conducteur décédé de polytraumatismes et sous influence du produit, les concentrations de mescaline étaient respectivement de $9,7 \mathrm{mg} / \mathrm{l}$ dans le sang, $70,8 \mathrm{mg} / \mathrm{kg}$ dans le foie et $1163 \mathrm{mg} / \mathrm{l}$ dans les urines.

Des observations de défénestration oי des homicides sous influence de mescaline ont pu être rapportés. Un article récent (6) relate l'homicide d'un Indien américain, consommateur de peyotl pour des raisons religieuses. Les concentrations de mescaline étaient respectivement de 2,95 mg/l (sang), 2,36 mg/l (humeur vitrée), $8,2 \mathrm{mg} / \mathrm{kg}$ (foie) et $2,2 \mathrm{mg} / \mathrm{kg}$ (encéphale).

\section{Conclusion}

L'usage de la mescaline sous forme cristalline ou à partir d'une couronne de peyotl semble rare en France. L'explosion du marché des designer drugs et l'utilisation exponentielle des rave drugs, dont le LSD, doivent rendre le toxicologue vigilant sur ce type de produit et devrait conduire à inclure la mescaline dans les protocoles analytiques de screening.

\section{Références}

1. Shulgin A., Shulgin A. Mescaline. In : Joy D., ed. PIHKAL A chemical love story. Berkeley : Transform Press, $1995 ; 702-7$.

2. Charalampous K.D., Walker K.E., Kinross-Wright J. Metabolic fate of mescaline in man, Psychopharmacologia $1966 ; 9$ : 48-63.

3. Demisch L., Kaczmarczyk P., Seiler N. 3,4,5-trimethoxybenzoic acid, a new mescaline metabolite in humans, Drug Met. Disp. 1978 ; 6 : 507-9.

4. Conti M., Zahra A., Antoine J., Pok Phak R., Viala A. Quelques aspects analytiques de la mescaline. J. Toxicol. Clin. Exp. $1990 ; 10$ : 385-94.

5. Reynolds P.C., Jindrich E.J. A mescaline associated fatality. J. Anal. Toxicol. $1985 ; 9$ : 183-4.

6. Henry J.L., Epley J., Rohrig T.P. The analysis and distribution of mescaline in postmortem tissues. J. Anal. Toxicol. $2003 ; 27: 381-2$. 
Annales de Toxicologie Analytique, vol. XVI, $n^{\circ}$ 1, 2004

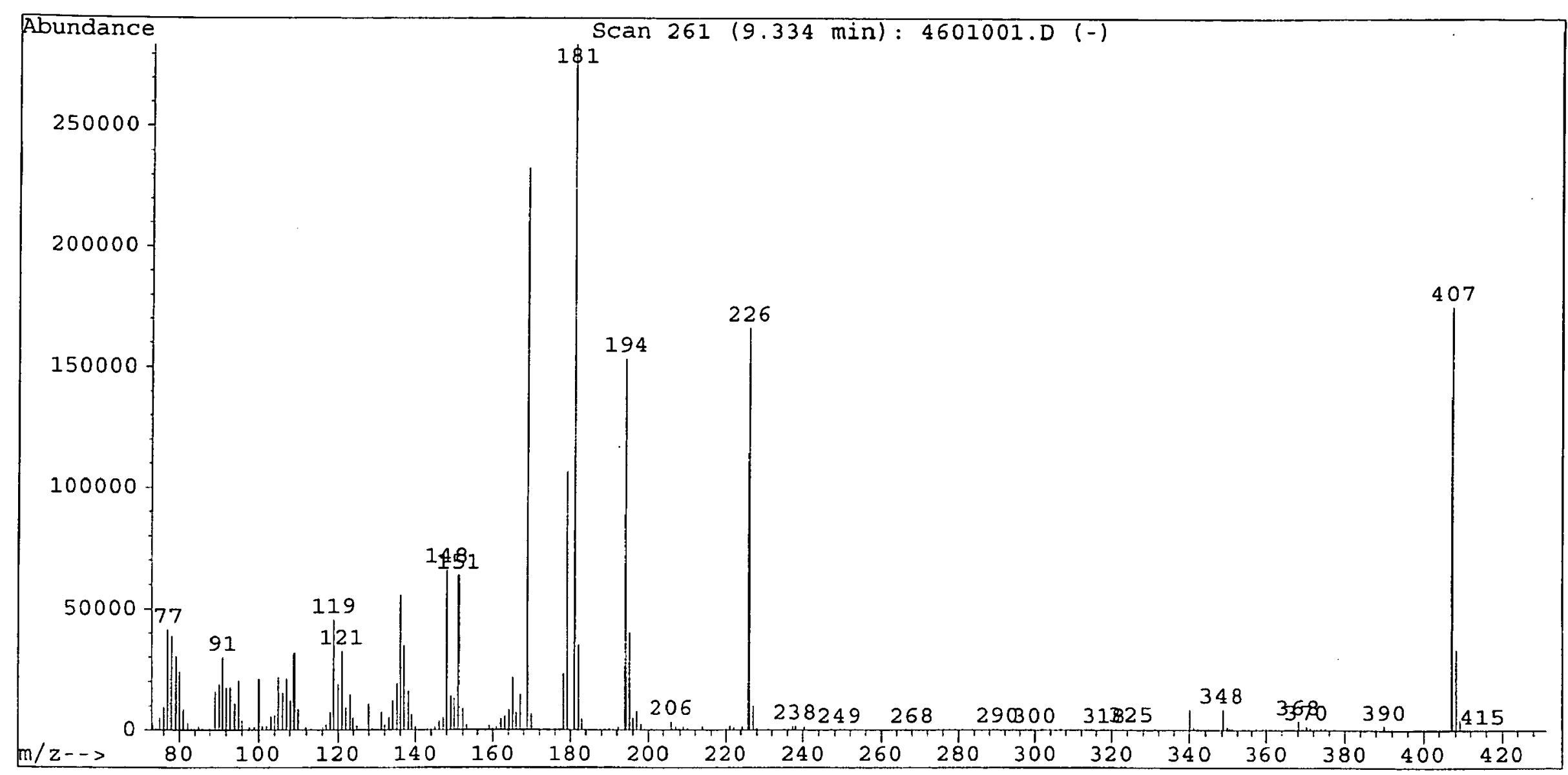

Figure 2 : Impact électronique de la mescaline dérivée par HFBA.

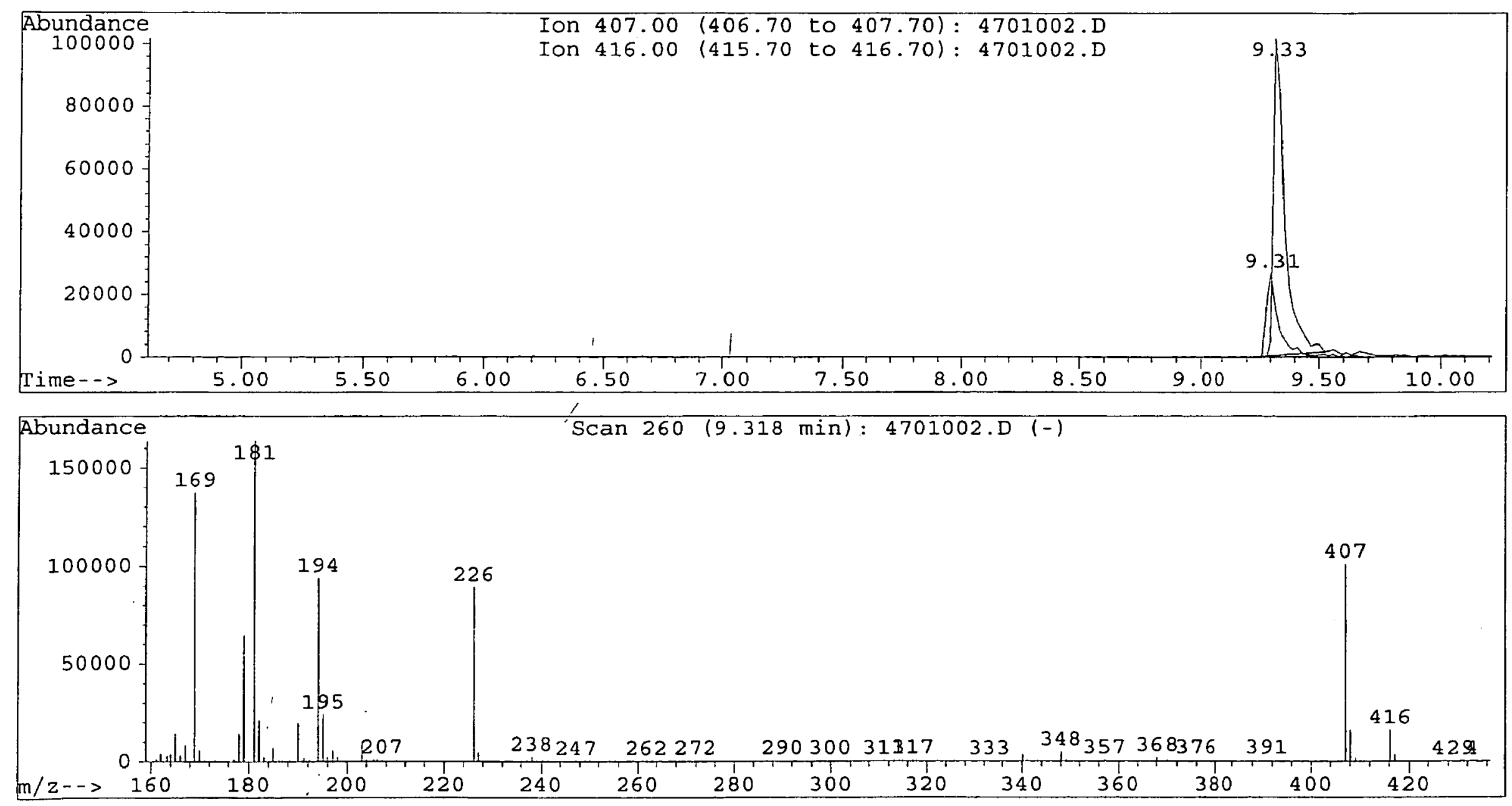

Figure 3 : En haut : Chromatogramme en mode SIM obtenu à partir d'un échantillon urinaire. La concentration de mescaline est de 0,89 $\mathrm{mg} / \mathrm{l}$.

En bas: Impact électronique de la mescaline dérivée par HFBA. A noter, la similitude des ions de la mescaline deutérée, à l'exclusion dẹ l'ion de quantification ( $\mathrm{m} / \mathrm{z} 407$ et 416). 